\title{
Optimization Problems over Unit-Distance Representations of Graphs
}

\author{
Marcel K. de Carli Silva* Levent Tunçel ${ }^{\dagger}$ \\ Department of Combinatorics and Optimization \\ University of Waterloo \\ Waterloo, Ontario, Canada \\ \{mksilva,ltuncel\}@math. uwaterloo.ca
}

Submitted: Jan 25, 2012; Accepted: Feb 14, 2013; Published: Feb 25, 2013

Mathematics Subject Classifications: 05C62, 90C35, 90C22

\begin{abstract}
We study the relationship between unit-distance representations and the Lovász theta number of graphs, originally established by Lovász. We derive and prove min-max theorems. This framework allows us to derive a weighted version of the hypersphere number of a graph and a related min-max theorem. Then, we connect to sandwich theorems via graph homomorphisms. We present and study a generalization of the hypersphere number of a graph and the related optimization problems. The generalized problem involves finding the smallest ellipsoid of a given shape which contains a unit-distance representation of the graph. Arbitrary positive semidefinite forms describing the ellipsoids yield NP-hard problems.
\end{abstract}

\section{Introduction}

Geometric representation of graphs is a beautiful area where combinatorial optimization, graph theory and semidefinite optimization meet and connect with many other research areas. In this paper, we start by studying geometric representations of graphs where each node is mapped to a point on a hypersphere so that each edge has unit length and the radius of the hypersphere is minimum. Lovász [15] proved that this graph invariant is related to the Lovász theta number of the complement of the graph via a simple but nonlinear equation. We show that this tight relationship leads to min-max theorems and

\footnotetext{
* Research of this author was supported in part by a Sinclair Scholarship, a Tutte Scholarship, Discovery Grants from NSERC, and by ONR research grant N00014-12-10049.

${ }^{\dagger}$ Research of this author was supported in part by a research grant from University of Waterloo, Discovery Grants from NSERC and by ONR research grant N00014-12-10049.
} 
to a "dictionary" to translate existing results about the theta function and its variants to the hypersphere representation setting and vice versa.

Based on our approach, we derive a weighted version of the hypersphere number of a graph and deduce related min-max theorems. Our viewpoint allows us to make new connections, strengthen some facts and correct some inaccuracies in the literature.

After observing that the hypersphere number of a graph is equal to the radius of the smallest Euclidean ball containing a unit-distance representation of the graph, we propose generalizations of the underlying optimization problems. Given a graph, the generalized optimization problem seeks the smallest ellipsoid of given shape which contains a unit-distance representation of the graph. We finally show that at this end of the new spectrum of unit-distance representations, arbitrary positive semidefinite forms describing the shapes of the ellipsoids yield NP-hard geometric representation problems.

\section{Preliminaries}

We denote the set of symmetric $n \times n$ matrices by $\mathbb{S}^{n}$, the set of symmetric $n \times n$ positive semidefinite matrices by $\mathbb{S}_{+}^{n}$, and the set of symmetric $n \times n$ positive definite matrices by $\mathbb{S}_{++}^{n}$. For a finite set $V$, the set of symmetric $V \times V$ matrices is denoted by $\mathbb{S}^{V}$, and the symbols $\mathbb{S}_{+}^{V}$ and $\mathbb{S}_{++}^{V}$ are defined analogously. Similarly, the set of orthogonal $n \times n$ matrices is denoted by $\mathbb{O}^{n}$, and for a finite set $V$, the set of orthogonal $V \times V$ matrices is denoted by $\mathbb{O}^{V}$. The unique positive semidefinite square root of $X \in \mathbb{S}_{+}^{V}$ is denoted by $X^{1 / 2}$. For $A, B \in \mathbb{S}^{n}$, we write $A \succeq B$ meaning $(A-B) \in \mathbb{S}_{+}^{n}$. Define an inner product on $\mathbb{S}^{n}$ by $\langle A, B\rangle:=\operatorname{Tr}(A B)$, where $\operatorname{Tr}(X):=\sum_{i=1}^{n} X_{i i}$ is the trace of $X \in \mathbb{R}^{n \times n}$. The linear map diag: $\mathbb{S}^{n} \rightarrow \mathbb{R}^{n}$ extracts the diagonal of a matrix; its adjoint is denoted by Diag.

For $A \in \mathbb{S}^{n}$, we denote by $\lambda^{\downarrow}(A) \in \mathbb{R}^{n}$ (resp., $\lambda^{\uparrow}(A) \in \mathbb{R}^{n}$ ) the vector of eigenvalues of $A$, with multiplicities, arranged in a nonincreasing (resp., nondecreasing) order, and we set $\lambda_{\max }(A):=\lambda_{1}^{\downarrow}(A)$ and $\lambda_{\min }(A):=\lambda_{1}^{\uparrow}(A)$.

The vector of all ones is denoted by $\bar{e}$. We abbreviate $[n]:=\{1, \ldots, n\}$. The notation $\|\cdot\|$ for a norm is the Euclidean norm unless otherwise specified. The convex hull of $S \subseteq \mathbb{R}^{n}$, denoted by $\operatorname{conv}(S)$, is the intersection of all convex subsets of $\mathbb{R}^{n}$ containing $S$. The set of nonnegative reals is denoted by $\mathbb{R}_{+}$. The set of positive reals is denoted by $\mathbb{R}_{++}$. Define the notations $\mathbb{Z}_{+}$and $\mathbb{Z}_{++}$analogously for integer numbers.

If $X$ and $Y$ are vector spaces, the direct sum of $X$ and $Y$ is the vector space $X \oplus Y$ : $=\{(x, y): x \in X, y \in Y\}$ with operations defined componentwise. An element $(x, y)$ of $X \oplus Y$ is also denoted by $x \oplus y$. If $A: X \rightarrow X$ and $B: Y \rightarrow Y$ are linear operators, then $A \oplus B$ is the linear operator $x \oplus y \in X \oplus Y \mapsto A x \oplus B y$. The nullspace of a linear operator $A: X \rightarrow X$ is denoted by $\operatorname{Null}(A)$.

Let $G$ be a graph. Its node set is $V(G)$ and its edge set is $E(G)$; when the graph $G$ is clear from the context, we just write $V$ for $V(G)$ and $E$ for $E(G)$. For $S \subseteq V$, the subgraph of $G$ induced by $S$, denoted by $G[S]$, is the subgraph of $G$ on $S$ whose edges are the edges of $G$ that have both ends in $S$. For $i \in V$, the neighbourhood of $i$, denoted by $N(i)$, is the set of nodes of $G$ adjacent to $i$. A cut-node of $G$ is a node $i \in V$ such 
that $G[V \backslash\{i\}]$ has more connected components than $H$. A block of $G$ is an inclusionwise maximal induced subgraph of $G$ with no cut-nodes.

For any function $f$ on graphs, we denote by $\bar{f}$ the function defined by $\bar{f}(G):=f(\bar{G})$ for every graph $G$, where $\bar{G}$ denotes the complement of $G$. In particular, $\bar{E}$ denotes the edge set of $\bar{G}$ when $G$ is clear from the context. For a graph $G$, we denote the clique number of $G$ by $\omega(G)$ and the chromatic number of $G$ by $\chi(G)$. The complete graph on $[n]$ is denoted by $K_{n}$.

For a graph $G$, the Laplacian of $G$ is the linear extension $\mathcal{L}_{G}: \mathbb{R}^{E} \rightarrow \mathbb{S}^{V}$ of the map $e_{\{i, j\}} \mapsto\left(e_{i}-e_{j}\right)\left(e_{i}-e_{j}\right)^{T}$ for every $\{i, j\} \in E$, where $e_{i}$ denotes the $i$ th unit vector. Laplacians arise naturally in spectral graph theory and spectral geometry; see [3].

\section{$3 \quad$ Hypersphere representations and the Lovász theta function}

Let $G$ be a graph. A unit-distance representation of $G$ is a function $u: V \rightarrow \mathbb{R}^{d}$ for some $d \geqslant 1$ such that $\|u(i)-u(j)\|=1$ whenever $\{i, j\} \in E$. A hypersphere representation of $G$ is a unit-distance representation of $G$ that is contained in a hypersphere centered at the origin, and the hypersphere number of $G$, denoted by $t(G)$, is the square of the smallest radius of a hypersphere that contains a unit-distance representation of $G$. The theta number of $G$ is defined by

$$
\vartheta(G):=\max \left\{\bar{e}^{T} X \bar{e}: \operatorname{Tr}(X)=1, X_{i j}=0 \forall\{i, j\} \in E, X \in \mathbb{S}_{+}^{V}\right\} .
$$

This parameter was introduced by Lovász in the seminal paper [14]; see also [8, 12] for further properties and alternative definitions.

Lovász [15, p. 23] noted the following formula relating $t$ and $\vartheta$ :

Theorem 3.1 ([15]). For every graph $G$, we have

$$
2 t(G)+1 / \bar{\vartheta}(G)=1 .
$$

We will show how the relation (3.2) can be used to better understand some of the properties of the theta number and the hypersphere number. This will allow us to obtain simpler proofs of some facts about the theta number and new results about hypersphere representations.

\subsection{Proof of Theorem 3.1}

We include a proof of Theorem 3.1 for the sake of completeness. We may formulate $t(G)$ as the SDP

$$
t(G)=\min \left\{t: \operatorname{diag}(X)=t \bar{e}, \mathcal{L}_{G}^{*}(X)=\bar{e}, X \in \mathbb{S}_{+}^{V}, t \in \mathbb{R}\right\} .
$$

Here, $\mathcal{L}_{G}^{*}$ is the adjoint of the Laplacian $\mathcal{L}_{G}$ of $G$. The dual of (3.3) is

$$
\max \left\{\bar{e}^{T} z: \operatorname{Diag}(y) \succeq \mathcal{L}_{G} z, \bar{e}^{T} y=1, y \in \mathbb{R}^{V}, z \in \mathbb{R}^{E}\right\} .
$$


Both (3.3) and (3.4) have Slater points, so SDP strong duality holds for this dual pair of SDPs, i.e., their optimal values coincide and both optima are attained. In particular, $t(G)$ is equal to (3.4). If we write an optimal solution $X^{*}$ of $(3.3)$ as $X^{*}=U U^{T}$, then $i \mapsto U^{T} e_{i}$ is a hypersphere representation of $G$ with squared radius $t(G)$.

Proof of Theorem 3.1. We can rewrite the dual (3.4) as

$$
t(G)=\max \left\{\frac{1}{2}\left\langle\bar{e} \bar{e}^{T}-I, Y\right\rangle: \bar{e}^{T} Y \bar{e}=1, Y_{i j}=0 \forall\{i, j\} \in \bar{E}(G), Y \in \mathbb{S}_{+}^{V}\right\}
$$

by taking $Y:=\operatorname{Diag}(y)-\mathcal{L}_{G}(z)$. Note that the objective value of a feasible solution $Y$ is $\frac{1}{2}\left\langle\bar{e} \bar{e}^{T}-I, Y\right\rangle=\frac{1}{2}(1-\operatorname{Tr}(Y))$. Thus, $t(G)=\frac{1}{2}(1-\hat{t}(G))$, where

$$
\hat{t}(G):=\min \left\{\operatorname{Tr}(Y): \bar{e}^{T} Y \bar{e}=1, Y_{i j}=0 \forall\{i, j\} \in \bar{E}(G), Y \in \mathbb{S}_{+}^{V}\right\} .
$$

It is easy to check that $\hat{t}(G) \bar{\vartheta}(G)=1$.

\subsection{Hypersphere and orthonormal representations of graphs}

Let $G$ be a graph. An orthonormal representation of $G$ is a function from $V$ to the unit hypersphere in $\mathbb{R}^{d}$ for some $d \geqslant 1$ that maps non-adjacent nodes to orthogonal vectors. It is known [15, sec. 4] that, if $u: V \rightarrow \mathbb{R}^{d}$ is a hypersphere representation of $G$ with squared radius $t \leqslant 1 / 2$, then the map

$$
q: i \mapsto \sqrt{2}[\sqrt{1 / 2-t} \oplus u(i)] \in \mathbb{R} \oplus \mathbb{R}^{d}
$$

is an orthonormal representation of $\bar{G}$. Define $\mathrm{TH}(G)$ as the set of all $x \in \mathbb{R}_{+}^{V}$ such that $\sum_{i \in V}\left(c^{T} p(i)\right)^{2} x_{i} \leqslant 1$ for every orthonormal representation $p: V \rightarrow \mathbb{R}^{d}$ of $G$ and unit vector $c \in \mathbb{R}^{d}$. Then $\vartheta(G)=\max \left\{\bar{e}^{T} x: x \in \mathrm{TH}(G)\right\}$.

The transformation (3.5) allows us to interpret Theorem 3.1 as strong duality for a nonlinear min-max relation:

Proposition 3.2. Let $G$ be a graph. For every hypersphere representation of $G$ with squared radius $t$ and every nonzero $x \in \mathrm{TH}(\bar{G})$, we have

$$
2 t+1 /\left(\bar{e}^{T} x\right) \geqslant 1
$$

with equality if and only if $t=t(G)$ and $\bar{e}^{T} x=\bar{\vartheta}(G)$.

Proof. Let $u: V \rightarrow \mathbb{R}^{d}$ be a hypersphere representation of $G$ with squared radius $t$. We may assume that $t<1 / 2$. Let $x \in \mathrm{TH}(\bar{G})$. Define an orthonormal representation $q$ of $\bar{G}$ from $p$ as in (3.5). Set $c:=1 \oplus 0 \in \mathbb{R} \oplus \mathbb{R}^{d}$. Then $(1-2 t) \bar{e}^{T} x=\sum_{i \in V}\left(c^{T} q(i)\right)^{2} x_{i} \leqslant 1$.

The equality case now follows from Theorem 3.1.

Proposition 3.2 shows that $\bar{\vartheta}(G)$ and elements from $\mathrm{TH}(\bar{G})$ are natural dual objects for $t(G)$ and hypersphere representations of $G$. In fact, using a well-known description of the elements of $\mathrm{TH}(\bar{G})$, we recover from Proposition 3.2 the following SDP-free purely geometric min-max relation: 
Corollary 3.3. Let $G$ be a graph. For every hypersphere representation of $G$ with squared radius $t$, every orthonormal representation $p: V \rightarrow \mathbb{R}^{d}$ of $G$, and every unit vector $c \in \mathbb{R}^{d}$ such that $c \notin p(V)^{\perp}$, we have

$$
2 t+\left[\sum_{i \in V}\left(c^{T} p(i)\right)^{2}\right]^{-1} \geqslant 1,
$$

with equality if and only if $t=t(G)$ and $\sum_{i \in V}\left(c^{T} p(i)\right)^{2}=\bar{\vartheta}(G)$.

\subsection{A Gallai-type identity}

The transformation (3.5) may be reversed as follows. Suppose that $q: V \rightarrow \mathbb{R}^{d}$ is an orthonormal representation of $\bar{G}$ such that, for some positive $\mu \in \mathbb{R}$ and some $u: V \rightarrow \mathbb{R}^{d-1}$, we have

$$
q(i)=\sqrt{2}\left[(2 \mu)^{-1 / 2} \oplus u(i)\right] \quad \forall i \in V .
$$

Then $u$ is a hypersphere representation of $G$ with squared radius $\frac{1}{2}(1-1 / \mu)$. We can use (3.5) and (3.6) to obtain an identity involving these objects.

Proposition 3.4. Let $G$ be a graph. Then

$$
2 t(G)+\max _{p, c} \min _{i \in V}\left(c^{T} p(i)\right)^{2}=1,
$$

where $p$ ranges over all orthonormal representations of $\bar{G}$ and $c$ over unit vectors of the appropriate dimension.

Proof. We first prove " $\leqslant$ " in (3.7). Let $p: V \rightarrow \mathbb{R}^{d}$ be an orthonormal representation of $\bar{G}$ and let $c \in \mathbb{R}^{d}$ be a unit vector. We will show that

$$
t(G) \leqslant \frac{1}{2}\left(1-\min _{i \in V}\left(c^{T} p(i)\right)^{2}\right) .
$$

It is well-known that there exists an orthonormal representation $q$ of $\bar{G}$ and a unit vector $d$ such that $\left(d^{T} q(j)\right)^{2}=\beta:=\min _{i \in V}\left(c^{T} p(i)\right)^{2}$ for all $j \in V$. If $\beta=0$, then $i \mapsto 2^{-1 / 2} e_{i} \in \mathbb{R}^{V}$ shows that $t(G) \leqslant 1 / 2$, so assume that $\beta>0$. We may assume that $d=e_{1}$ and $d^{T} q(i) \geqslant 0$ for every $i \in V$. Now use (3.6) with $\mu=1 / \beta$ to get a hypersphere representation $u$ of $G$ from $q$ with squared radius $\frac{1}{2}(1-\beta)$. This proves (3.8).

Next we prove " $\geqslant$ " in (3.7). Let $u: V \rightarrow \mathbb{R}^{d}$ be a hypersphere representation of $G$ with squared radius $t(G)$. Build an orthonormal representation $q$ of $\bar{G}$ as in (3.5) and pick $c:=1 \oplus 0 \in \mathbb{R} \oplus \mathbb{R}^{d}$. Then $\left(c^{T} q(i)\right)^{2}=1-2 t(G)$ for every $i \in V$.

(The reciprocal of the second term of the sum on the LHS of (3.7) was used as the original definition of $\bar{\vartheta}(G)$ by Lovász [14].)

Note that (3.7) does not provide a good characterization of either $t(G)$ or the maximization problem on the LHS of (3.7). In this sense, Proposition 3.4 is akin to Gallai's identities for graphs [16, Lemmas 1.0.1 and 1.0.2]. 


\subsection{Unit-distance representations in hyperspheres and balls}

For a graph $G$, let $t_{b}(G)$ be the square of the smallest radius of an Euclidean ball that contains a unit-distance representation of $G$. This parameter is also mentioned by Lovász [15, Proposition 4.1].

To formulate $t_{b}(G)$ as an SDP, replace the constraint $\operatorname{diag}(X)=t \bar{e}$ in (3.3) by $\operatorname{diag}(X) \leqslant t \bar{e}$. The resulting SDP and its dual have Slater points, so SDP strong duality holds, i.e., both optima are attained and the optimal values coincide.

Evidently, $t_{b}(G) \leqslant t(G)$ for every graph $G$. In fact, equality holds:

Theorem 3.5. For every graph $G$, we have $t_{b}(G)=t(G)$.

If we mimic the proof of Theorem 3.1 for $t_{b}(G)$, we find that

$$
2 t_{b}(G)+1 / \overline{\vartheta_{b}}(G)=1,
$$

where $\vartheta_{b}(G)$ is defined by adding the constraint $X \bar{e} \geqslant 0$ to the SDP (3.1). Thus, by (3.2) and (3.9), Theorem 3.5 is equivalent to the fact that $\vartheta_{b}(G)=\vartheta(G)$ for every graph $G$. This follows from next result [6, Proposition 9] (this was pointed out to the first author by Fernando Mário de Oliveira Filho):

Proposition 3.6 ([6]). Let $\mathbb{K} \subseteq \mathbb{S}^{n}$ be such that $\operatorname{Diag}(h) X \operatorname{Diag}(h) \in \mathbb{K}$ whenever $X \in \mathbb{K}$ and $h \in \mathbb{R}_{+}^{n}$. If $\hat{X}$ is an optimal solution for $\max \left\{\bar{e}^{T} X \bar{e}: \operatorname{Tr}(X)=1, X \in \mathbb{K} \cap \mathbb{S}_{+}^{n}\right\}$, then $\operatorname{diag}(\hat{X})=\mu \hat{X} \bar{e}$ for some positive $\mu \in \mathbb{R}$.

Proof of Theorem 3.5. Since $\vartheta(G)$ is a relaxation of $\vartheta_{b}(G)$, we have $\vartheta_{b}(G) \leqslant \vartheta(G)$. To prove the reverse inequality, let $\hat{X}$ be an optimal solution for (3.1). By Proposition 3.6, we have $\hat{X} \bar{e}=\mu^{-1} \operatorname{diag}(\hat{X}) \geqslant 0$ for some $\mu>0$. Hence $\hat{X}$ is feasible for the SDP that defines $\vartheta_{b}(G)$, whence $\vartheta_{b}(G) \geqslant \vartheta(G)$.

\subsection{Hypersphere proofs of $\vartheta$ facts}

The formula (3.2) relating $t(G)$ and $\bar{\vartheta}(G)$ allows us to infer some basic facts about the theta number from a geometrically simpler viewpoint.

Theorem 3.7 (The Sandwich Theorem [14]). For any graph $G$, we have

$$
\omega(G) \leqslant \bar{\vartheta}(G) \leqslant \chi(G) .
$$

By Theorem 3.1 and the fact that $\bar{\vartheta}\left(K_{n}\right)=n$ for every $n \geqslant 1$, the Sandwich Theorem is equivalent to the inequalities $t\left(K_{\omega(G)}\right) \leqslant t(G) \leqslant t\left(K_{\chi(G)}\right)$ for every graph $G$. The first inequality is obvious: if $H$ is a subgraph of $G$, then $t(H) \leqslant t(G)$. The second one is also obvious: if $u:[\ell] \rightarrow \mathbb{R}^{d}$ is a hypersphere representation of $K_{\ell}$ and $c: V \rightarrow[\ell]$ is a colouring of $G$, then $u \circ c$ is a hypersphere representation of $G$. This hints at a strong connection with graph homomorphisms, which we will look at more closely in Section 4.

Lovász [15, p. 34] mentions that a graph $G$ is bipartite if and only if $\bar{\vartheta}(G) \leqslant 2$. The less obvious of the implications may be easily proved by showing that $\bar{\vartheta}\left(C_{n}\right)>2$ for every 
odd cycle $C_{n}$. However, we find that the following proof using hypersphere representations gives a more enlightening geometric interpretation. By Theorem 3.1, we must show that $t(G) \leqslant 1 / 4$ if and only if $G$ is bipartite. If $G$ is bipartite, then $G$ has a hypersphere representation with radius $1 / 2$ even in $\mathbb{R}^{1}$. Suppose $G$ has a hypersphere representation with radius $\leqslant 1 / 2$. The only pairs of points at distance 1 in a hypersphere of radius $1 / 2$ are the pairs of antipodal points, so $G$ is bipartite.

Given graphs $G$ and $H$ with $V(G) \cap V(H)=\varnothing$, the direct sum of $G$ and $H$ is the graph $G+H$ with node set $V(G) \cup V(H)$ and edge set $E(G) \cup E(H)$. It is proved in [12] that $\bar{\vartheta}(G+H)=\max \{\bar{\vartheta}(G), \bar{\vartheta}(H)\}$. By Theorem 3.1, this is equivalent to the geometrically obvious equation $t(G+H)=\max \{t(G), t(H)\}$. In particular, $t(G)=$ $\max \{t(C): C$ a component of $G\}$. More generally, $t(G)=\max \{t(B): B$ a block of $G\}$. This follows from the next result, where $G \cup H$ denotes the graph with node set $V(G) \cup V(H)$ and edge set $E(G) \cup E(H)$ and $G \cap H$ denotes the graph with node set $V(G) \cap V(H)$ and edge set $E(G) \cap E(H)$.

Proposition 3.8. Let $G$ be a graph, and suppose $G=G_{1} \cup G_{2}$ for graphs $G_{1}$ and $G_{2}$, with $G_{1} \cap G_{2}$ a complete graph. Then

$$
t(G)=\max \left\{t\left(G_{1}\right), t\left(G_{2}\right)\right\} \quad \text { and } \quad \bar{\vartheta}(G)=\max \left\{\bar{\vartheta}\left(G_{1}\right), \bar{\vartheta}\left(G_{2}\right)\right\} .
$$

Proof. By Theorem 3.1, it suffices to prove that $t(G)=\max \left\{t\left(G_{1}\right), t\left(G_{2}\right)\right\}$. Clearly ' $\geqslant$ ' holds in the desired equation. Assume $t\left(G_{1}\right) \geqslant t\left(G_{2}\right)$. Since the feasible region of (3.3) is convex and contains $(\bar{X}, \bar{t}):=\frac{1}{2}(I, 1)$, there are hypersphere representations $u$ and $v$ of $G_{1}$ and $G_{2}$, respectively, both with squared radius $t\left(G_{1}\right)$. We may assume that the images of $u$ and $v$ live in the same Euclidean space. Since $G_{1} \cap G_{2}$ is a complete graph, there is an orthogonal matrix $Q$ such that $Q v(i)=u(i)$ for every $i \in V\left(G_{1} \cap G_{2}\right)$. If we glue the hypersphere representation $i \mapsto Q v(i)$ of $G_{2}$ with $u$, we get a hypersphere representation of $G$ with squared radius $t\left(G_{1}\right)$.

This behavior of $t$ and $\bar{\vartheta}$ with respect to clique sums is shared by many other graph parameters, e.g., $\omega, \chi$, the Hadwiger number (the size of the largest clique minor), and the graph invariant $\lambda$ introduced in [9].

Proposition 3.8 and Theorem 3.5 imply the following purely geometric result:

Corollary 3.9. Let $G$ be a graph, and suppose $G=G_{1} \cup G_{2}$ for graphs $G_{1}$ and $G_{2}$, with $G_{1} \cap G_{2}$ a complete graph. For $i \in\{1,2\}$, let $u_{i}$ be a unit-distance representation of $G_{i}$ contained in an Euclidean ball of radius $r_{i}$. Then there is a unit-distance representation of $G$ contained in an Euclidean ball of radius $\max \left\{r_{1}, r_{2}\right\}$.

The proof contains an algorithm to build the desired unit-distance representation of $G$. However, whereas one would expect such an algorithm to provide a geometric construction from $u_{1}$ and $u_{2}$, the one presented essentially needs to solve an SDP, and it may ignore $u_{1}$ and $u_{2}$ altogether.

Using basic properties of Laplacians, we can prove the following behaviour of $t$ and $\bar{\vartheta}$ with respect to edge contraction: 
Proposition 3.10. Let $G$ be a graph and let $e=\{i, j\} \in E$. If $(\bar{y}, \bar{z})$ is an optimal solution for (3.4), then $\bar{z}_{e} \geqslant t(G)-t(G / e)$. If $\bar{X}$ is an optimal solution for (3.1) applied to $\bar{\vartheta}(G)$, then $\bar{\vartheta}(G) \leqslant\left(2 \bar{X}_{i j}+1\right) \bar{\vartheta}(G / e)$.

Proof. See Appendix A.

Finally, using basic properties about the intersection of two hyperspheres, we can prove a property of $\bar{\vartheta}$ that is shared by the parameters $\omega, \chi$, and the fractional chromatic number $\chi^{*}$. The proof is based on [11, Lemma 4.3].

Proposition 3.11. Let $G$ be a graph and $i \in V$ with $N(i) \neq \varnothing$. Then

$$
t(G[N(i)]) \leqslant 1-1 /[4 t(G)] \text { and } \bar{\vartheta}(G) \geqslant \bar{\vartheta}(G[N(i)])+1 .
$$

Proof. See Appendix A.

\subsection{A weighted version}

For $w \in \mathbb{R}_{+}^{V}$, define $\vartheta(G, w)$ by replacing the objective function in (3.1) by $\sqrt{w}^{T} X \sqrt{w}$, where $(\sqrt{w})_{i}:=\sqrt{w_{i}}$ for every $i \in V$. It is natural to define a weighted hypersphere number $t(G, w)$ so that it satisfies a natural generalization of equation (3.2), namely, $2 t(G, w)+1 / \vartheta(\bar{G}, w)=1$ whenever $w \neq 0$. By using the proof of Theorem 3.1 as a guide, we arrive at the definition:

$$
\begin{aligned}
t(G, w):=\min & t \\
& \operatorname{diag}(X)=\frac{1}{2} \bar{e}+\left(t-\frac{1}{2}\right) w, \\
& \mathcal{L}_{G}^{*}(X)=\bar{e}+\left(t-\frac{1}{2}\right) \mathcal{L}_{G}^{*}\left(\sqrt{w} \sqrt{w}^{T}\right), \\
& X \in \mathbb{S}_{+}^{V}, t \in \mathbb{R} .
\end{aligned}
$$

This SDP and its dual have Slater points, so SDP strong duality holds.

Even though we cannot offer a nice direct interpretation for this definition of $t(G, w)$, by construction, we can generalize Proposition 3.2:

Theorem 3.12. Let $G$ be a graph and $w \in \mathbb{R}_{+}^{V} \backslash\{0\}$. Then, for every feasible solution $(X, t)$ of (3.10) and every nonzero $x \in \mathrm{TH}(\bar{G})$, we have

$$
2 t+1 /\left(w^{T} x\right) \geqslant 1,
$$

with equality if and only if $(X, t)$ is optimal for (3.10) and $w^{T} x=\vartheta(\bar{G}, w)$.

Proof. We may assume that $t<1 / 2$. Write $X=P^{T} P$ for some $[d] \times V$ matrix $P$, and define $p: V \rightarrow \mathbb{R}^{d}$ by $p: i \mapsto P e_{i}$. The map $q: i \mapsto \sqrt{2}\left[\sqrt{w_{i}(1 / 2-t)} \oplus p(i)\right] \in \mathbb{R} \oplus \mathbb{R}^{d}$ is an orthonormal representation of $\bar{G}$. Put $c:=1 \oplus 0 \in \mathbb{R} \oplus \mathbb{R}^{d}$. Then $(1-2 t) w^{T} x=$ $\sum_{i \in V}\left(c^{T} q(i)\right)^{2} x_{i} \leqslant 1$.

The equality case now follows by construction. 
If $w \in \mathbb{Z}_{+}^{V}$, it can be shown that $t(G, w)=t\left(G^{w}\right)$, where $G^{w}$ is obtained from $G$ by replacing each node $i$ by a clique $G_{i}$ on $w_{i}$ nodes; if $\{i, j\} \in E$, then every node in $G_{i}$ is adjacent in $G^{w}$ to every node in $G_{j}$.

In fact, every feasible solution $(\bar{X}, \bar{t})$ of $(3.10)$ encodes a hypersphere representation of $G^{w}$ with squared radius $\bar{t}$. Indeed, write $\bar{X}=P^{T} P$ for some $[d] \times V$ matrix $P$, and define $p: i \mapsto P e_{i}$. For $i \in V$, let $q_{i}: V\left(G_{i}\right) \rightarrow \mathbb{R}^{d_{i}}$ be a hypersphere representation of $G_{i}$ with squared radius $t\left(G_{i}\right)=\frac{1}{2}\left(1-1 / w_{i}\right)$. Define $u: V\left(G^{w}\right) \rightarrow \mathbb{R}^{d} \oplus\left(\bigoplus_{i \in V} \mathbb{R}^{d_{i}}\right)$ as follows.

For $k \in V\left(G_{i}\right)$, set $u(k)$ to be the vector whose block in $\mathbb{R}^{d}$ is $w_{i}^{-1 / 2} p(i)$ and whose block in $\mathbb{R}^{d_{i}}$ is $q_{i}(k)$; all other blocks of $u(k)$ are zero. Then $u$ is a hypersphere representation of $G^{w}$ with squared radius $\bar{t}$.

\section{Graph homomorphisms and sandwich theorems}

Let $G$ and $H$ be graphs. A homomorphism from $G$ to $H$ is a function $f: V(G) \rightarrow V(H)$ such that $\{f(i), f(j)\} \in E(H)$ whenever $\{i, j\} \in E(G)$. If there is a homomorphism from $G$ to $H$, we write $G \rightarrow H$.

Note that $t(G) \leqslant t(H)$ whenever $G \rightarrow H$. Indeed, if $f$ is a homomorphism from $G$ to $H$ and $v$ is a hypersphere representation of $H$, then $v \circ f$ is a hypersphere representation of $G$. This combines with the graph-theoretic observation that $K_{\omega(G)} \rightarrow G \rightarrow K_{\chi(G)}$ to yield $t\left(K_{\omega(G)}\right) \leqslant t(G) \leqslant t\left(K_{\chi(G)}\right)$, which by Theorem 3.1 is equivalent to the Sandwich Theorem 3.7.

Motivated by this, we call a real-valued graph invariant $f$ hom-monotone if $f(G) \leqslant f(H)$ whenever $G \rightarrow H$ and the following "nondegeneracy" condition holds: there is a nondecreasing function $g: \operatorname{Im}(f) \rightarrow \mathbb{R}$ such that $g\left(f\left(K_{n}\right)\right)=n$ for every integer $n \geqslant 1$. Using these properties for an arbitrary graph $G$ and the fact that $K_{\omega(G)} \rightarrow G \rightarrow K_{\chi(G)}$, we get $f\left(K_{\omega(G)}\right) \leqslant f(G) \leqslant f\left(K_{\chi(G)}\right)$, and thus

$$
\omega(G) \leqslant g(f(G)) \leqslant \chi(G) .
$$

(See [2] for a similar use of these ideas.) We point out that hom-monotonicity cannot recover strong Sandwich Theorems which state that $\omega(G) \leqslant \bar{\vartheta}(G) \leqslant \chi^{*}(G)$ since this inequality fails to hold for the hom-monotone invariant $\chi$.

The function $g(x):=1 /(1-2 x)$ is non-decreasing on $[0,1 / 2) \supseteq \operatorname{Im}(t)$, so $t$ is hommonotone, and we recover from (4.1) the Sandwich Theorem 3.7.

The reason why $t$ satisfies the first condition of hom-monotonicity roughly comes from the fact that the constraints for the SDP (3.3) of $t$ are "uniform" for the edges, i.e., all edges are treated in the same way. We are thus led to define other SDPs of the same type. One such example is the parameter $t_{b}$. However, as we have seen in Theorem 3.5, this parameter is equal to $t$. Now define

$$
t^{\prime}(G):=\min \left\{t: \operatorname{diag}(X)=t \bar{e}, \mathcal{L}_{G}^{*}(X) \geqslant \bar{e}, X \in \mathbb{S}_{+}^{V}, t \in \mathbb{R}\right\} .
$$

Clearly, $t^{\prime}(G) \leqslant t(G)$ for every graph $G$, and it is easy to see that equality holds if $G$ is node-transitive. In particular, $t^{\prime}\left(K_{n}\right)=t\left(K_{n}\right)$ for every $n$. Thus, the function $g(x):=1 /(1-2 x)$ proves that $t^{\prime}$ is hom-monotone. 
Using (4.1) and $t^{\prime}(G) \leqslant t(G)$, we obtain $\omega(G) \leqslant g\left(t^{\prime}(G)\right) \leqslant g(t(G)) \leqslant \chi(G)$ for every graph $G$. If we mimic the proof of Theorem 3.1 for $t^{\prime}(G)$, we find that $2 t^{\prime}(G)+1 / \overline{\vartheta^{\prime}}(G)=1$, where $\vartheta^{\prime}(G)$ is defined by adding the constraint $X \geqslant 0$ to $(3.1)$, i.e., $g\left(t^{\prime}(G)\right)=\vartheta^{\prime}(G)$ is the graph parameter introduced in [17] and [20].

Let $\operatorname{dim}(G)$ be the minimum $d \geqslant 0$ such that there is a unit-distance representation of $G$ in $\mathbb{R}^{d}$; consider $\mathbb{R}^{0}:=\{0\}$. As before, $G \rightarrow H$ implies $\operatorname{dim}(G) \leqslant \operatorname{dim}(H)$. Since $\operatorname{dim}\left(K_{n}\right)=n-1$, the function $g(x):=x+1$ shows that dim is hom-monotone, so $\omega(G) \leqslant \operatorname{dim}(G)+1 \leqslant \chi(G)$. However, we will see later that computing $\operatorname{dim}(G)$ is NP-hard. (A similar parameter was introduced in [4].)

Define $\operatorname{dim}_{h}(G)$ similarly as $\operatorname{dim}(G)$ but for hypersphere representations of $G$ with squared radius $\leqslant 1 / 2$ and $\operatorname{dim}_{o}(G)$ for orthonormal representations of $\bar{G}$. Such parameters are also hom-monotone. Clearly $\operatorname{dim}(G) \leqslant \operatorname{dim}_{h}(G)$ for every graph $G$, but strict inequality occurs for the Mosers spindle (see Figure 1 and the proof of Theorem 5.4). Since (3.5) shows that $\operatorname{dim}_{o}(G) \leqslant \operatorname{dim}_{h}(G)+1$ and [14] shows that $\bar{\vartheta}(G) \leqslant \operatorname{dim}_{o}(G)$, these parameters are related by $\omega(G) \leqslant \overline{\vartheta^{\prime}}(G) \leqslant \bar{\vartheta}(G) \leqslant \operatorname{dim}_{o}(G) \leqslant \operatorname{dim}_{h}(G)+1 \leqslant \chi(G)$. In particular, by $(3.2)$, we find that $\operatorname{dim}_{h}(G) \geqslant 2 t(G) /(1-2 t(G))$. Also $\operatorname{dim}_{h}(G) \leqslant \chi(G)-1 \leqslant \Delta(G)$, where $\Delta(G)$ is the maximum degree of $G$. In fact, by Brooks' Theorem, $\operatorname{dim}_{h}(G) \leqslant \Delta(G)-1$ when $G$ is connected but not complete nor an odd cycle.

\subsection{Hypersphere representations and vector colourings}

The following relaxation of graph colouring was introduced in [11]. Let $G$ be a graph. For a real number $k \geqslant 1$, a vector $k$-colouring of $G$ is a function $p$ from $V$ to the unit hypersphere in $\mathbb{R}^{d}$ for some $d \geqslant 1$ such that $\langle p(i), p(j)\rangle \leqslant-1 /(k-1)$ whenever $\{i, j\} \in E$; we consider the fraction to be $-\infty$ if $k=1$, so the only graphs that have a vector 1-colouring are the graphs with no edges.

A vector $k$-colouring $p$ of $G$ is strict if $\langle p(i), p(j)\rangle=-1 /(k-1)$ for every $\{i, j\} \in E$, and a strict vector $k$-colouring $p$ of $G$ is strong if $\langle p(i), p(j)\rangle \geqslant-1 /(k-1)$ whenever $\{i, j\} \in \bar{E}(G)$.

The vector chromatic number of $G$ is the smallest $k \geqslant 1$ for which there exists a vector $k$-colouring of $G$, and the strict vector chromatic number and strong vector chromatic number are defined analogously.

It is easy to show (see, e.g., [13]) that the vector chromatic number of $G$ is $\overline{\vartheta^{\prime}}(G)$, the strict vector chromatic number of $G$ is $\bar{\vartheta}(G)$, and the strong vector chromatic number of $G$ is $\overline{\vartheta^{+}}(G)$, known as Szegedy's number [22], where $\vartheta^{+}(G)$ is defined by replacing the constraints $X_{i j}=0$ for every $\{i, j\} \in E$ in (3.1) by $X_{i j} \leqslant 0$ for every $\{i, j\} \in E$.

Here, we note that a scaling map yields a correspondence between these variations of vector colourings and unit-distance representations, provided that the graph $G$ has at least one edge.

Let $p$ be a strict vector $k$-colouring of $G$. Then the map $i \mapsto t p(i)$, where $t^{2}=\frac{1}{2}(1-1 / k)$, is a hypersphere representation of $G$ with squared radius $t$. Conversely, if $q$ is a hypersphere representation of $G$ with squared radius $t<1 / 2$, then the map $i \mapsto t^{-1 / 2} q(i)$, is a strict vector $k$-colouring of $G$, where $k=1 /(1-2 t)$. This correspondence shows that 
$t(G)=\frac{1}{2}\left(1-1 / \chi_{v}(G)\right)$, where $\chi_{v}(G)$ denotes the strict vector chromatic number of $G$.

The same scaling maps as above yield correspondences between vector $k$-colourings and the geometric representations arising from the graph invariant $t^{\prime}$, and also between strong vector $k$-colourings and geometric representations arising from the graph invariant

$$
\begin{aligned}
t^{+}(G):=\min & t \\
& \operatorname{diag}(X)=t \bar{e}, \\
& X_{i i}-2 X_{i j}+X_{j j}=1, \quad \forall\{i, j\} \in E, \\
& X_{i i}-2 X_{i j}+X_{j j} \leqslant 1, \quad \forall\{i, j\} \in \bar{E}, \\
& X \in \mathbb{S}_{+}^{V}, t \in \mathbb{R} .
\end{aligned}
$$

Note however, that the parameter $t^{+}$does not fit into the framework of hom-monotone graph invariants since the SDP (4.3) has non-edge constraints.

We point out here that, while these equivalences between variants of vector chromatic number and variants of theta number are easy to prove, they are not as widely known as they should be. For instance, in [1] it is shown that the vector chromatic number $\chi_{v}^{\prime}(G)$ of $G$ satisfies

$$
\chi_{v}^{\prime}(G) \geqslant \max \left\{1-\frac{\lambda_{\max }(B)}{\lambda_{\min }(B)}: B \in \mathcal{A}_{G}, B \geqslant 0\right\},
$$

where $\mathcal{A}_{G}$ denotes the set of all weighted adjacency matrices of $G$, i.e., all symmetric $V \times V$ matrices $A$ such that $A_{i j} \neq 0 \Longrightarrow\{i, j\} \in E$. However, since $\chi_{v}^{\prime}(G)=\overline{\vartheta^{\prime}}(G)$, it is possible to adapt the proof of the Hoffman bounds for $\vartheta(G)$ (see, e.g., [12, Corollary 33]) to show that (4.4) actually holds with equality.

Also, in [18, Remark 3.1] it is reported that a certain graph $G$ has vector chromatic number strictly smaller than its strict vector chromatic number, and that it was unknown whether some such graph existed. However, this statement about the vector chromatic numbers is equivalent to $\overline{\vartheta^{\prime}}(G)<\bar{\vartheta}(G)$, and the existence of graphs satisfying this strict inequality was already known as far back as 1979 (see [20]).

We also mention that one of the characterizations of $\vartheta^{\prime}(G)$ in [7] and [5] is inaccurate. Define an obtuse representation of a graph $G$ to be a map $p: V \rightarrow \mathbb{R}^{d}$ for some $d \geqslant 1$ such that

(i) $\|p(i)\|=1$ for every $i \in V$, and

(ii) $\langle p(i), p(j)\rangle \leqslant 0$ for every $\{i, j\} \in \bar{E}$.

In $[7$, Theorem 1] and [5, p. 133] it is claimed that

$$
\vartheta^{\prime}(G)=\min _{p, c} \max _{i \in V} \frac{1}{\left(c^{T} p(i)\right)^{2}},
$$

where $p$ ranges over obtuse representations of $G$ and $c$ ranges over unit vectors of appropriate dimension. Let $G$ be a $2 n$-partite graph with color classes $C_{1}, \ldots, C_{2 n}$ such that $\omega(G)=2 n$. Thus, $\vartheta^{\prime}(\bar{G}) \geqslant \omega(G)=2 n$. Let $p(j):=e_{i} \in \mathbb{R}^{n}$ for every $j \in C_{i}$ and $i \in[n]$, and $p(j):=-e_{i} \in \mathbb{R}^{n}$ for every $j \in C_{n+i}$ and $i \in[n]$. Set $c:=n^{-1 / 2} \bar{e} \in \mathbb{R}^{n}$. By (4.5), we get $\vartheta^{\prime}(\bar{G}) \leqslant n$, a contradiction. 
Now we show how to fix the formula (4.5). Given an obtuse representation $p: V \rightarrow \mathbb{R}^{d}$ of a graph $G$, we say that a vector $c \in \mathbb{R}^{d}$ is consistent with $p$ if $c^{T} p(i) \geqslant 0$ for every $i \in V$. The next result is a Gallai-type identity involving $t^{\prime}(G)$, parallel to Proposition 3.4 for $t(G)$.

Proposition 4.1. Let $G$ be a graph. Then

$$
2 t^{\prime}(G)+\max _{p, c} \min _{i \in V}\left(c^{T} p(i)\right)^{2}=1
$$

where $p$ ranges over all obtuse representations of $\bar{G}$ and $c$ over unit vectors consistent with $p$.

The proof of Proposition 4.1 is analogous to the proof of Proposition 3.4, with the following slight adjustments. In the notation of the proof of (3.8), the vector $d$ may be chosen to be consistent with the obtuse representation $q$, so we do not need to replace any of the $q(i)$ 's by their opposites.

Corollary 4.2. Let $G$ be a graph. Then $\vartheta^{\prime}(G)$ is given by (4.5), where $p$ ranges over obtuse representations of $G$ and $c$ ranges over unit vectors consistent with $p$.

Proof. This follows from Proposition 4.1 and the formula $2 t^{\prime}(G)+1 / \overline{\vartheta^{\prime}}(G)=1$.

\section{Unit-distance representations in ellipsoids}

The graph parameter $t_{b}$ encodes the problem of finding the smallest Euclidean ball that contains a unit-distance representation of a given graph. In this section, we study graph parameters that encode the problem of finding the smallest ellipsoid of a given shape that contains a unit-distance representation of a given graph.

Let $G$ be a graph. In Section 3.4, we defined $t_{b}(G)$ as the minimum infinity-norm of the vector $\left(u_{i}^{T} u_{i}\right)_{i \in V}$ over all unit-distance representations $u$ of $G$, where we are using the notation $u_{i}:=u(i)$. It is natural to replace the vector $\left(u_{i}^{T} u_{i}\right)_{i \in V}$ in the objective function of the previous optimization problem with the vector $\left(u_{i}^{T} A u_{i}\right)_{i \in V}$ for some fixed $A \in \mathbb{S}_{++}^{d}$. The resulting optimization problem corresponds to finding the minimum squared radius $t$ such that the ellipsoid $\left\{x \in \mathbb{R}^{d}: x^{T} A x \leqslant t\right\}$ contains a unit-distance representation of $G$.

We are thus led to define, for every graph $G$, every $A \in \mathbb{S}_{+}^{d}$ for some $d \geqslant 1$, and every $p \in[1, \infty]$, the number $\mathcal{E}_{p}(G ; A)$ as the infimum of $\left\|\left(u_{i}^{T} A u_{i}\right)_{i \in V}\right\|_{p}$ as $u$ ranges over all unit-distance representations of $G$ in $\mathbb{R}^{d}$, or equivalently,

$$
\mathcal{E}_{p}(G ; A):=\inf \left\{\left\|\operatorname{diag}\left(U A U^{T}\right)\right\|_{p}: \mathcal{L}_{G}^{*}\left(U U^{T}\right)=\bar{e}, U \in \mathbb{R}^{V \times[d]}\right\} .
$$

Note that we allow $A$ to be singular.

Since the feasible region in (5.1) is invariant under right-multiplication by matrices in $\mathbb{O}^{d}$, we have $\mathcal{E}_{p}(G ; A)=\mathcal{E}_{p}\left(G ; Q A Q^{T}\right)$ for every $Q \in \mathbb{O}^{d}$. In particular, $\mathcal{E}_{p}(G ; \cdot)$ is a spectral function. 
Let us derive some basic properties of the optimal solutions of $\mathcal{E}_{p}(G ; A)$. First, we prove that if $\mathcal{E}_{p}(G ; A)$ is finite then the corresponding optimal geometric representation exists. The first observation towards this goal is that, if $G$ is connected, then the maximum distance between any pair of points in every unit-distance representation is at most $(|V|-1)$.

Theorem 5.1. Let $G$ be a graph. Let $A \in \mathbb{S}_{+}^{d}$ for some $d \geqslant 1$ and let $p \in[1, \infty]$. If $\mathcal{E}_{p}(G ; A)<+\infty$, then there exists a matrix $U \in \mathbb{R}^{V \times[d]}$ such that $\mathcal{L}_{G}^{*}\left(U U^{T}\right)=\bar{e}$ and $\left\|\operatorname{diag}\left(U A U^{T}\right)\right\|_{p}=\mathcal{E}_{p}(G ; A)$.

Proof. We may assume that $G$ is connected. (If not, it suffices to focus on the component $H$ of $G$ with $\mathcal{E}_{p}(H ; A)=\mathcal{E}_{p}(G ; A)$.) We may further assume $A=\operatorname{Diag}(a)$, where $a=$ $\lambda^{\downarrow}(A) \neq 0$. So, there exists a largest $k \in[d]$ so that $a_{k} \neq 0$. Let $A^{\prime}:=\operatorname{Diag}\left(a_{1}, \ldots, a_{k}\right)$. Throughout this proof, let $P: \mathbb{R}^{d} \rightarrow \mathbb{R}^{k}$ denote the projection onto the first $k$ components, i.e., $P\left(x_{1}, \ldots, x_{d}\right)^{T}=\left(x_{1}, \ldots, x_{k}\right)^{T}$, and let $Q: \mathbb{R}^{d} \rightarrow \mathbb{R}^{d-k}$ denote the projection onto the last $d-k$ components. Note that $A=P^{T} A^{\prime} P$ and $A^{\prime} \succeq a_{k} I$.

Let $M \in \mathbb{R}$ such that $\mathcal{E}_{p}(G ; A) \leqslant M$. Fix $j \in V$ arbitrarily. We claim that the following constraints may be added to the RHS of (5.1) without changing its optimal value:

$$
\begin{aligned}
\left\|P U^{T} e_{i}\right\|_{2}^{2} & \leqslant B:=(M+1) / a_{k} \quad \text { for every } i \in V, \\
Q U^{T} e_{j} & =0 .
\end{aligned}
$$

Let us see why this proves the theorem. Let $U \in \mathbb{R}^{V \times[d]}$ be feasible for (5.1) and satisfy (5.2) and (5.3). Let $i \in V$ be arbitrary. Since the columns of $U^{T}$ form a unitdistance representation of $G$, the distance in $G$ between $i$ and $j$ is an upper bound for $\left\|U^{T} e_{i}-U^{T} e_{j}\right\|_{2}$. Hence, $\left\|U^{T} e_{i}\right\|_{2} \leqslant\left\|U^{T} e_{j}\right\|_{2}+|V|=\left\|P U^{T} e_{j}\right\|_{2}+|V| \leqslant B^{1 / 2}+|V|$. Thus, the new feasible region is compact and we will be done.

First, we prove that the constraints (5.2) may be added to (5.1) without changing the optimal value. Suppose $U \in \mathbb{R}^{V \times[d]}$ violates (5.2) for some $i \in V$. Then we find that $\left\|\operatorname{diag}\left(U A U^{T}\right)\right\|_{p} \geqslant e_{i}^{T} U A U^{T} e_{i}=e_{i}^{T} U P^{T} A^{\prime} P U^{T} e_{i} \geqslant e_{i}^{T} U P^{T}\left(a_{k} I\right) P U^{T} e_{i}=$ $a_{k}\left\|P U^{T} e_{i}\right\|_{2}^{2}>M+1 \geqslant \mathcal{E}_{p}(G ; A)+1$, so $U$ may be discarded from the feasible set of (5.2).

Next, we add the constraint (5.3). Let $U \in \mathbb{R}^{V \times[d]}$ be feasible for (5.1) and satisfy (5.2). Define $X \in \mathbb{R}^{V \times[d]}$ by setting $P X^{T} e_{i}:=P U^{T} e_{i}$ for every $i \in V$ and $Q X^{T} e_{i}:=Q U^{T} e_{i}-$ $Q U^{T} e_{j}$ for every $i \in V$. Hence, $X$ is feasible for (5.1) and satisfies (5.2) and (5.3). Moreover, $\operatorname{diag}\left(X A X^{T}\right)=\operatorname{diag}\left(U A U^{T}\right)$. This completes the proof.

A geometrically pleasing, intuitive conjecture is that a suitably defined notion of a "centre" of an optimal representation of every graph must coincide with the centre of the ellipsoid. The next result takes a step along this direction by refining the previous theorem.

Theorem 5.2. Let $G$ be a graph. Let $A \in \mathbb{S}_{+}^{d}$ for some $d \geqslant 1$ and let $p \in[1, \infty]$. If $\mathcal{E}_{p}(G ; A)<+\infty$, then there is a unit-distance representation $u: V \rightarrow \mathbb{R}^{d}$ of $G$ such that $\left\|\left(u_{i}^{T} A u_{i}\right)_{i \in V}\right\|_{p}=\mathcal{E}_{p}(G ; A)$ and $0 \in \operatorname{conv}(u(V))$. 
Proof. We use the same assumptions and notation defined in the first paragraph of the proof of Theorem 5.1. Let $u: V \rightarrow \mathbb{R}^{d}$ be a feasible solution for $\mathcal{E}_{p}(G ; A)$. Let $\mathcal{U}$ be the set of all unit-distance representations of $G$ of the form $i \in V \mapsto u_{i}+r$ for some vector $r \in \mathbb{R}^{d}$ such that $\operatorname{Pr}=0$. Note that if $k=d$, then $\mathcal{U}$ is a singleton. Clearly, every element of $\mathcal{U}$ has the same objective value as $u$. We will show that if there does not exist some element $v \in \mathcal{U}$ such that $0 \in \operatorname{conv}(v(V))$, then $\mathcal{E}_{p}(G ; A)<\left\|\left(u_{i}^{T} A u_{i}\right)_{i \in V}\right\|_{p}$. Then this theorem will follow from Theorem 5.1.

So, assume that $0 \notin M:=\bigcup_{v \in \mathcal{U}} \operatorname{conv}(v(V))$. Since $M=\operatorname{conv}(u(V))+\operatorname{Null}(P)$ is a polyhedron and $0 \notin M$, there exists $h \in \mathbb{R}^{d}$ and $\alpha>0$ such that $h^{T} v_{i} \geqslant \alpha$ for every $v \in \mathcal{U}$ and $i \in V$. Note that $Q h=0$ since for each $j \in\{k+1, \ldots, d\}$ the linear function $h^{T} u_{i}+t h_{j}=h^{T}\left(u_{i}+t e_{j}\right)$ of $t$ is bounded below by $\alpha$. Thus,

$$
h^{T} u_{i} \geqslant \alpha>0, \quad \forall i \in V \quad \text { and } \quad h \in \operatorname{Im}(A) .
$$

Let $x \in \mathbb{R}^{d}$ such that $A x=h$ and let $s:=\varepsilon x$, where $\varepsilon>0$ will be chosen later. Define $v: V \rightarrow \mathbb{R}^{d}$ by $v_{i}:=u_{i}-s$. Let $i \in V$. Then $v_{i}^{T} A v_{i}=u_{i}^{T} A u_{i}-2 \varepsilon h^{T} u_{i}+\varepsilon^{2} x^{T} A x$. Hence $v_{i}^{T} A v_{i}<u_{i}^{T} A u_{i}$ if and only if $2 \varepsilon h^{T} u_{i}>\varepsilon^{2} x^{T} A x$. Thus, we will be done if we can find $\varepsilon>0$ such that $2 h^{T} u_{i}>\varepsilon x^{T} A x$. Since $h^{T} u_{i} \geqslant \alpha>0$, such $\varepsilon$ exists. This shows that, for some choice of $\varepsilon>0$, we have $v_{i}^{T} A v_{i}<u_{i}^{T} A u_{i}$ for every $i \in V$, whence $\mathcal{E}_{p}(G ; A) \leqslant\left\|\left(v_{i}^{T} A v_{i}\right)_{i \in V}\right\|_{p}<\left\|\left(u_{i}^{T} A u_{i}\right)_{i \in V}\right\|_{p}$.

The next result shows that it is not very interesting to use arbitrarily large prescribed embedding dimension $d$ :

Theorem 5.3. Let $G$ be a graph. Let $A \in \mathbb{S}_{+}^{d}$ for some $d \geqslant 1$ and let $p \in[1, \infty]$. If $k \in[d]$ is such that $\mathcal{E}_{p}(G ; A)$ has an optimal solution $u: V \rightarrow \mathbb{R}^{d}$ with $\operatorname{dim}(\operatorname{span}(u(V))) \leqslant k$, then

$$
\mathcal{E}_{p}(G ; A)=\mathcal{E}_{p}\left(G ; B_{k}\right)
$$

where $B_{k}:=\operatorname{Diag}\left(\lambda_{1}^{\uparrow}(A), \ldots, \lambda_{k}^{\uparrow}(A)\right)$. In particular, $\mathcal{E}_{p}(G ; A)=\mathcal{E}_{p}\left(G ; B_{n-1}\right)$ if $d \geqslant n-1$.

Proof. We may assume that $A=\operatorname{Diag}(a)$ where $a=\lambda^{\uparrow}(A)$. Note that $B:=B_{k}=$ $\operatorname{Diag}\left(a_{1}, \ldots, a_{k}\right)$. The proof of ' $\leqslant$ ' in $(5.5)$ is immediate by appending extra zero coordinates to an optimal solution of $\mathcal{E}_{p}(G ; B)$.

To prove ' $\geqslant$,' let $u: V \rightarrow \mathbb{R}^{d}$ be an optimal solution for $\mathcal{E}_{p}(G ; A)$ such that $\operatorname{span}(u(V))$ has dimension $k$. Then, there exists $Q \in \mathbb{O}^{d}$ such that, for each $i \in V$, the final $d-k$ coordinates of $Q u_{i}$ are zero. Let $v_{i} \in \mathbb{R}^{k}$ be obtained from $Q u_{i}$ by dropping the final $d-k$ (zero) coordinates. If $C \in \mathbb{S}_{+}^{k}$ is the principal submatrix of $Q A Q^{T}$ indexed by $[k]$, then $\left(v_{i}^{T} C v_{i}\right)_{i \in V}=\left(u_{i}^{T} A u_{i}\right)_{i \in V}$. Hence, $\mathcal{E}_{p}(G ; A)=\left\|\left(u_{i}^{T} A u_{i}\right)_{i \in V}\right\|_{p}=\left\|\left(v_{i}^{T} C v_{i}\right)_{i \in V}\right\|_{p} \geqslant \mathcal{E}_{p}(G ; C)$. By interlacing of eigenvalues, $\lambda^{\uparrow}(C) \geqslant \lambda^{\uparrow}(B)$. Hence, $\mathcal{E}_{p}(G ; A) \geqslant \mathcal{E}_{p}(G ; C) \geqslant \mathcal{E}_{p}(G ; B)$.

It follows from Theorem 5.2 that $\mathcal{E}_{p}(G ; A)=\mathcal{E}_{p}\left(G ; B_{n-1}\right)$ if $d \geqslant n-1$.

It is clear that $\mathcal{E}_{p}(G ; A)=0$ if and only if $\operatorname{dim}(G) \leqslant \operatorname{dim}(\operatorname{Null}(A))$. So deciding whether $\operatorname{dim}(G) \leqslant k$ for any fixed $k$ reduces to computing $\mathcal{E}_{p}(G ; A)$ for any $p \in[1, \infty]$ where $A$ is a matrix of nullity $k$. It is easy to see that the former decision problem is NP-hard (see [10, Theorem 4]). We give below a shorter proof. 


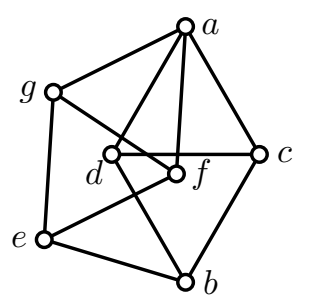

Figure 1: The Mosers spindle; see [21].

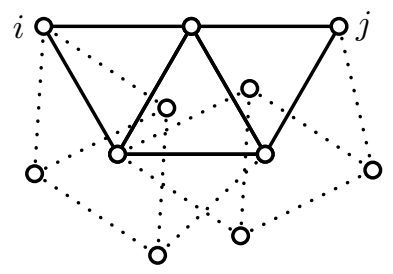

Figure 2: The gadget graph $H$.

Theorem 5.4 ([10]). The problem of deciding whether $\operatorname{dim}(G) \leqslant 2$ for a given input graph $G$ is NP-hard.

Proof. Let $k$ be a fixed positive integer. Saxe [19] showed that the following problem is NP-hard: given an input graph $G$ and $\ell: E \rightarrow \mathbb{R}_{+}$, decide whether there exists $u: V \rightarrow \mathbb{R}^{k}$ such that $\|u(i)-u(j)\|=\ell_{\{i, j\}}$ for every $\{i, j\} \in E$. Saxe showed that the problem remains NP-hard even if we require $\ell(E) \subseteq\{1,2\}$.

We will show a polynomial-time reduction from the above problem with $k=2$ and $\ell(E) \subseteq\{1,2\}$ to the problem of deciding whether $\operatorname{dim}(G) \leqslant 2$. It suffices to show how we can replace any edge of the input graph $G$ which is required to be embedded as a line segment of length 2 by some gadget graph $H$ so that every unit-distance representation of $H$ in $\mathbb{R}^{2}$ maps two specified nodes of $H$ to points at distance 2 .

Consider the graph $M$ known as the Mosers spindle shown in Figure 1. The subgraph of $M$ induced by $\{a, b, c, d\}$ has exactly two unit-distance representations in $\mathbb{R}^{2}$ modulo rigid motions: one of them as displayed in Figure 1, and the other one maps nodes $a$ and $b$ to the same point. We claim that, in any unit-distance representation $u$ of $M$ in $\mathbb{R}^{2}$, the nodes $a$ and $b$ are not mapped to the same point. Suppose otherwise. Since the points $u(e), u(f), u(g)$ are at distance 1 from $u(a)=u(b)$ and from each other, $u$ shows that $\operatorname{dim}\left(K_{4}\right) \leqslant 2$, whereas clearly $\operatorname{dim}\left(K_{4}\right) \geqslant 3$.

Let $H$ be the gadget shown in Figure 2, which consists of two copies of $M$ sharing a triangle (some edges of $M$ are drawn in dots for the sake of ease of visualization). Then, every unit-distance representation of $H$ in $\mathbb{R}^{2}$ maps the nodes $i$ and $j$ to points at distance 2. Thus, if we replace the corresponding edges $\{i, j\}$ of the input graph $G$ by $H$, we obtain a graph $G^{\prime}$ such that $\operatorname{dim}\left(G^{\prime}\right) \leqslant 2$ if and only if $G$ can be embedded in $\mathbb{R}^{2}$ with the prescribed edge lengths. 
It follows from Theorem 5.4 that, for any fixed $p \in[1, \infty]$, the problem of computing $\mathcal{E}_{p}(G ; A)$ for an input graph $G$ and $A \in \mathbb{S}_{+}^{V}$ is NP-hard. Hence the graph parameter $t_{b}=t$ is in a sense on the borderline of tractability.

\subsection{The extreme cases $p \in\{1, \infty\}$}

For every matrix $U \in \mathbb{R}^{V \times V}$, if we set $X:=U U^{T}$, then there exists an orthogonal $V \times V$ matrix $Q$ such that $U^{T}=Q X^{1 / 2}$. Hence, if $A \in \mathbb{S}_{+}^{V}$, then

$$
\begin{array}{cl}
\mathcal{E}_{p}(G ; A)=\inf & \left\|\operatorname{diag}\left(X^{1 / 2} Q^{T} A Q X^{1 / 2}\right)\right\|_{p} \\
& \mathcal{L}_{G}^{*}(X)=\bar{e} \\
& X \in \mathbb{S}_{+}^{V}, Q \in \mathbb{O}^{V} .
\end{array}
$$

When $p=1$, the objective function in (5.6) is $\operatorname{Tr}\left(Q^{T} A Q X\right)=\left\langle Q^{T} A Q, X\right\rangle$ so we can write

$$
\mathcal{E}_{1}(G ; A)=\inf _{Q \in \mathbb{O}^{V}} t_{Q^{T} A Q}(G)
$$

where $t_{W}(G)$ is defined for any $W \in \mathbb{S}^{V}$ as the SDP

$$
t_{W}(G):=\inf \left\{\langle W, X\rangle: \mathcal{L}_{G}^{*}(X)=\bar{e}, X \in \mathbb{S}_{+}^{V}\right\} .
$$

Proposition 5.5. Let $G$ be a connected graph and let $W \in \mathbb{S}^{V}$. Then $t_{W}(G)$ is finite if and only if $\bar{e}^{T} W \bar{e}>0$ or $W \bar{e}=0$. Moreover, whenever $t_{W}(G)$ is finite, both (5.8) and its dual SDP have optimal solutions and their optimal values coincide.

The parameter $t_{W}(G)$ thus underlies the parameters $\mathcal{E}_{1}(G ; A)$ as well as the hypersphere number $t(G)$, since (3.4) shows that

$$
t(G)=\min \left\{t_{\operatorname{Diag}(y)}(G): \bar{e}^{T} y=1, y \in \mathbb{R}^{V}\right\} .
$$

If $X$ is feasible in (5.8) for $G=K_{n}$, then $X$ is completely determined by its diagonal entries. Using this fact, it is easy to prove that the feasible region of (5.8) for $G=K_{n}$ is

$$
\left\{X \in \mathbb{S}_{+}^{n}: \mathcal{L}_{K_{n}}^{*}(X)=\bar{e}\right\}=\left\{\left(y \bar{e}^{T}+\bar{e} y^{T}+2 I\right) / 4:\|\bar{e}\|\|y\| \leqslant \bar{e}^{T} y+2, y \in \mathbb{R}^{n}\right\} .
$$

Using a second-order cone programming formulation, we can show that

$$
2 t_{W}\left(K_{n}\right)= \begin{cases}\operatorname{Tr}(W)-\frac{\|W \bar{e}\|^{2}}{\bar{e}^{T} W \bar{e}} & \text { if } \bar{e}^{T} W \bar{e}>0 \\ \operatorname{Tr}(W) & \text { if } W \bar{e}=0 \\ -\infty & \text { otherwise. }\end{cases}
$$

Let us use (5.7) and (5.10) to compute $\mathcal{E}_{1}(G ; A)$. Let $A \in \mathbb{S}_{+}^{n}$ be nonzero. Since $Q \bar{e} \notin \operatorname{Null}(A)$ for some $Q \in \mathbb{O}^{n}$, it follows from (5.7) and (5.10) that

$$
2 \mathcal{E}_{1}\left(K_{n} ; A\right)=\operatorname{Tr}(A)-\sup \left\{\frac{\left\|Q^{T} A Q \bar{e}\right\|^{2}}{\bar{e}^{T} Q^{T} A Q \bar{e}}: Q \bar{e} \notin \operatorname{Null}(A), Q \in \mathbb{O}^{n}\right\} .
$$


The supremum may be replaced by $\sup \left\{\left(h^{T} A^{2} h\right) /\left(h^{T} A h\right): h \in \operatorname{Null}(A)^{\perp}\right\}$, which is easily seen to be $\lambda_{\max }(A)$. This implies with Theorem 5.3 that

$$
\mathcal{E}_{1}\left(K_{n} ; A\right)= \begin{cases}\frac{1}{2} \sum_{i=1}^{n-1} \lambda_{i}^{\uparrow}(A) & \text { if } A \in \mathbb{S}_{+}^{d} \text { with } d \geqslant n-1 \\ +\infty & \text { otherwise. }\end{cases}
$$

For the other extreme $p=\infty$, the first property of hom-monotonicity holds. More precisely, let $\left(a_{n}\right)_{n \in \mathbb{Z}_{++}}$be a nondecreasing sequence of positive reals. Define $A_{n}:=$ $\operatorname{Diag}\left(a_{1}, \ldots, a_{n}\right)$ for every $n \in \mathbb{Z}_{++}$. Then,

$$
G \rightarrow H \Longrightarrow \mathcal{E}_{\infty}\left(G ; A_{n}\right) \leqslant \mathcal{E}_{\infty}\left(H ; A_{n}\right) .
$$

We do not know whether the graph invariant $\mathcal{E}_{\infty}$ satisfies the second property of hommonotonicity. In fact, we do not know an analytical formula to compute $\mathcal{E}_{\infty}\left(K_{n} ; A\right)$ in terms of $A$. However, we have such a formula for an infinite family of complete graphs, as we now describe. Let $H$ be an $n \times n$ Hadamard matrix, i.e., $H$ is $\{ \pm 1\}$-valued and $H^{T} H=n I$. We may assume that $H$ has the form $H^{T}=\left[\begin{array}{ll}\bar{e} & L^{T}\end{array}\right]$. Then $L^{T} L=n I-\bar{e} \bar{e}^{T}$, so $\frac{1}{2 n} \mathcal{L}_{K_{n}}^{*}\left(L^{T} L\right)=\bar{e}$, i.e., the map $i \mapsto(2 n)^{-1 / 2} L e_{i}$ is a unit-distance representation of $K_{n}$. This map is called a Hadamard representation of $K_{n}$.

Theorem 5.6. Let $n \in \mathbb{Z}_{++}$such that there exists an $n \times n$ Hadamard matrix. Then, for any $p \in[1, \infty]$ and diagonal $A \in \mathbb{S}_{+}^{n-1}$, every Hadamard representation of $K_{n}$ is an optimal solution for $\mathcal{E}_{p}\left(K_{n} ; A\right)$.

Proof. The objective value of the Hadamard representation $\bar{L}$ of $K_{n}$ in the optimization problem $\mathcal{E}_{p}\left(K_{n} ; A\right)$ is $\left[\frac{\operatorname{Tr}(A)}{2 n}\right]\|\bar{e}\|_{p}$. Thus, $\bar{L}$ is optimal for $p=1$ by (5.11). From the inequality $\|x\|_{1} \leqslant n\|x\|_{\infty}$ we get $\mathcal{E}_{\infty}\left(K_{n} ; A\right) \geqslant \frac{1}{n} \mathcal{E}_{1}\left(K_{n} ; A\right)$, which shows that $\bar{L}$ is optimal for $p=\infty$. Therefore, $\bar{L}$ is optimal for every $p \in[1, \infty]$.

It is natural to lift a Hadamard representation $h$ of $K_{n}$ to obtain a frugal feasible solution for $\mathcal{E}\left(K_{n+1} ; A\right)$. The image of $h$ is an $(n-1)$-dimensional simplex $\Delta$. If $v$ is a vertex of an $n$-dimensional simplex whose opposite facet is $\Delta$, then the line segment $L$ joining $v$ to the barycenter of $\Delta$ is the shortest line segment joining $v$ to $\Delta$. It makes sense to align $L$ with the most expensive axis, i.e., the one corresponding to $\lambda_{\max }(A)$. Suppose $A=\operatorname{Diag}(a)$ and $\|a\|_{\infty}=a_{n}$. We thus obtain a unit-distance representation $u$ of $K_{n+1}$ in $\mathbb{R}^{n}$ of the form

$$
u(i):= \begin{cases}h(i) \oplus \alpha, & \text { if } i \in[n] \\ 0 \oplus\left[\alpha+\left(\frac{n+1}{2 n}\right)^{1 / 2}\right], & \text { if } i=n+1 .\end{cases}
$$

By optimizing the shift parameter $\alpha$, we obtain the following upper bound:

Proposition 5.7. Let $n \in \mathbb{Z}_{++}$such that there exists an $n \times n$ Hadamard matrix. If $A \in \mathbb{S}_{+}^{n}$, then

$$
\mathcal{E}_{\infty}\left(K_{n+1} ; A\right) \leqslant \frac{\operatorname{Tr}(A)}{2(n+1)}+\frac{\left(\operatorname{Tr}(A)-n \lambda_{\max }(A)\right)^{2}}{8 n(n+1) \lambda_{\max }(A)} .
$$

Equality holds for $n=2$ if $A \succ 0$.

The proof of equality for $n=2$ involves the obvious parametrization of $\mathbb{O}^{2}$ and basic trigonometry. 


\section{References}

[1] Y. Bilu. Tales of Hoffman: three extensions of Hoffman's bound on the graph chromatic number. J. Combin. Theory Ser. B, 96(4):608-613, 2006.

[2] P. J. Cameron, A. Montanaro, M. W. Newman, S. Severini, and A. Winter. On the quantum chromatic number of a graph. Electron. J. Combin., 14(1):Research Paper 81, 15 pp. (electronic), 2007.

[3] F. R. K. Chung. Spectral graph theory, volume 92 of CBMS Regional Conference Series in Mathematics. Published for the Conference Board of the Mathematical Sciences, Washington, DC, 1997.

[4] P. Erdős, F. Harary, and W. T. Tutte. On the dimension of a graph. Mathematika, 12:118-122, 1965.

[5] A. Galtman. Spectral characterizations of the Lovász number and the Delsarte number of a graph. J. Algebraic Combin., 12(2):131-143, 2000.

[6] D. Gijswijt. Matrix Algebras and Semidefinite Programming Techniques for Codes. PhD thesis, University of Amsterdam, 2005.

[7] M. X. Goemans. Semidefinite programming in combinatorial optimization. Math. Programming, 79(1-3, Ser. B):143-161, 1997. Lectures on mathematical programming (ismp97) (Lausanne, 1997).

[8] M. Grötschel, L. Lovász, and A. Schrijver. Geometric algorithms and combinatorial optimization, volume 2 of Algorithms and Combinatorics. Springer-Verlag, Berlin, second edition, 1993.

[9] H. van der Holst, M. Laurent, and A. Schrijver. On a minor-monotone graph invariant. J. Combin. Theory Ser. B, 65(2):291-304, 1995.

[10] B. Horvat, J. Kratochvíl, and T. Pisanski. On the computational complexity of degenerate unit distance representations of graphs. In Combinatorial algorithms, volume 6460 of Lecture Notes in Comput. Sci., pages 274-285. Springer, Heidelberg, 2011.

[11] D. Karger, R. Motwani, and M. Sudan. Approximate graph coloring by semidefinite programming. J. ACM, 45(2):246-265, 1998.

[12] D. E. Knuth. The sandwich theorem. Electron. J. Combin., 1:Article 1, approx. 48 pp. (electronic), 1994.

[13] M. Laurent and F. Rendl. Semidefinite programming and integer programming. In Handbook on Discrete Optimization, pages 393-514. Elsevier B. V., Amsterdam, 2005.

[14] L. Lovász. On the Shannon capacity of a graph. IEEE Trans. Inform. Theory, 25(1):1-7, 1979.

[15] L. Lovász. Semidefinite programs and combinatorial optimization. In Recent advances in algorithms and combinatorics, pages 137-194. Springer, New York, 2003. 
[16] L. Lovász and M. D. Plummer. Matching theory, volume 121 of North-Holland Mathematics Studies. North-Holland Publishing Co., Amsterdam, 1986. Annals of Discrete Mathematics, 29.

[17] R. J. McEliece, E. R. Rodemich, and H. C. Rumsey, Jr. The Lovász bound and some generalizations. J. Combin. Inform. System Sci., 3(3):134-152, 1978.

[18] P. Meurdesoif. Strengthening the Lovász $\theta(\bar{G})$ bound for graph coloring. Math. Program., 102(3, Ser. A):577-588, 2005.

[19] J. B. Saxe. Two papers on graph embedding problems. Technical Report CMU-CS80-102, Department of Computer Science, Carnegie-Mellon University, 1980.

[20] A. Schrijver. A comparison of the Delsarte and Lovász bounds. IEEE Trans. Inform. Theory, 25(4):425-429, 1979.

[21] A. Soifer. The mathematical coloring book. Springer, New York, 2009. Mathematics of coloring and the colorful life of its creators, With forewords by Branko Grünbaum, Peter D. Johnson, Jr. and Cecil Rousseau.

[22] M. Szegedy. A note on the theta number of Lovász and the generalized Delsarte bound. In Proceedings of the 35th Annual IEEE Symposium on Foundations of Computer Science, 1994.

\section{A Some Omitted Proofs}

Proof of Proposition 3.10. Let $(\bar{y}, \bar{z})$ be an optimal solution for (3.4). We will construct a feasible solution for (3.4) applied to $G / e$ with objective value $t(G)-\bar{z}_{e}$. Assume $e=\{a, b\}$ and $V^{\prime}:=V(G / e)=V \backslash\{b\}$, so we are denoting the contracted node of $G / e$ by $a$. Let $P$ be the $V^{\prime} \times V$ matrix defined by $P:=e_{a} e_{b}^{T}+\sum_{i \in V^{\prime}} e_{i} e_{i}^{T}$. Then $P \mathcal{L}_{G}(\bar{z}) P^{T}=\mathcal{L}_{G / e}(\hat{z})$, where $\hat{z} \in \mathbb{R}^{E(G / e)}$ is obtained from $\bar{z}$ as follows. In taking the contraction $G / e$ from $G$, immediately after we identify the ends of $e$, but before we remove resulting parallel edges, there are at most two edges between each pair of nodes of $G / e$, as we assume that $G$ is simple. If there is exactly one edge between nodes $i$ and $j$, we just set $\hat{z}_{\{i, j\}}:=\bar{z}_{\{i, j\}}$. If there are two edges joining nodes $i$ and $j$, say $f$ and $f^{\prime}$, we put $\hat{z}_{\{i, j\}}:=\bar{z}_{f}+\bar{z}_{f^{\prime}}$.

Similarly, if we define $\hat{y}: V^{\prime} \rightarrow \mathbb{R}$ by putting $\hat{y}_{i}:=\bar{y}_{i}$ for $i \in V^{\prime} \backslash\{a\}$ and $\hat{y}_{a}:=\bar{y}_{a}+\bar{y}_{b}$, then $P \operatorname{Diag}(\bar{y}) P^{T}=\operatorname{Diag}(\hat{y})$. Since $P \mathbb{S}_{+}^{V} P^{T} \subseteq \mathbb{S}_{+}^{V^{\prime}}$, we see that $(\hat{y}, \hat{z})$ is a feasible solution for (3.4) applied to $G / e$, and its objective value is $\hat{z}(E(G / e))=\bar{z}(E)-\bar{z}_{e}$.

To prove the inequality involving $\bar{\vartheta}(G)$, use (3.2) together with its proof to see that $\bar{X}$ corresponds to an optimal solution $(\bar{y}, \bar{z})$ for $(3.4)$ with $\bar{X} / \bar{\vartheta}(G)=\operatorname{Diag}(\bar{y})-\mathcal{L}_{G}(\bar{z})$, so $\bar{y}_{e}=\bar{X}_{i j} / \bar{\vartheta}(G)$.

Proof of Proposition 3.11. By Theorem 3.1, it suffices to show $t(G[N(i)]) \leqslant 1-1 /[4 t(G)]$. Let $p: V \rightarrow \mathbb{R}^{d}$ be a hypersphere representation of $G$ with squared radius $t:=t(G)$. We may assume that $p(i)=t^{1 / 2} e_{1}$. For every $j \in N(i)$, we have $1=\|p(i)-p(j)\|^{2}=$ $\|p(i)\|^{2}+\|p(j)\|^{2}-2\langle p(i), p(j)\rangle=2 t-2 t^{1 / 2}[p(j)]_{1}$. Hence, $[p(j)]_{1}=(2 t-1) /\left(2 t^{1 / 2}\right)=: \beta$ for every $j \in N(i)$. Define the following hypersphere representation of $G[N(i)]$ : for each 
$j \in N(i)$, let $q(j)$ be obtained from $p(j)$ by dropping the first coordinate. The squared radius of the resulting hypersphere representation is $t-\beta^{2}=1-1 /(4 t)$.

Proof of (5.9). Let $X \in \mathbb{S}^{V}$. Then $\mathcal{L}_{K_{n}}^{*}(X)=\bar{e}$ if and only if $4 X=y \bar{e}^{T}+\bar{e} y^{T}+2 I$ for some $y \in \mathbb{R}^{V}$; for the 'only if' part, use $y:=2 \operatorname{diag}(X)-\bar{e}$.

Let $y \in \mathbb{R}^{V}$. The smallest eigenvalue of $y \bar{e}^{T}+\bar{e} y^{T}$ is $\bar{e}^{T} y-\|\bar{e}\|\|y\|$. Thus, we have $y \bar{e}^{T}+\bar{e} y^{T}+2 I \succeq 0$ if and only if $\|\bar{e}\|\|y\| \leqslant \bar{e}^{T} y+2$.

Proof of (5.10). Assume first that $W=\operatorname{Diag}(w)$ for some $w \in \mathbb{R}^{n}$. By Proposition 5.5, finiteness of $t_{W}\left(K_{n}\right)$ implies $\bar{e}^{T} w>0$ or $w=0$. Assume the former. By (5.9),

$$
2 t_{W}\left(K_{n}\right)=\bar{e}^{T} w+\min \left\{w^{T} y:\|\bar{e}\| y_{0}-\bar{e}^{T} y=2, y_{0} \oplus y \in \mathrm{SOC}_{n}\right\},
$$

where $\mathrm{SOC}_{n}:=\left\{y_{0} \oplus y \in \mathbb{R} \oplus \mathbb{R}^{n}:\|y\| \leqslant y_{0}\right\}$. The second-order cone program on the RHS of (A.1) has $\bar{y}_{0} \oplus \bar{y}:=\left(2+\|\bar{e}\|^{2}\right) /\|\bar{e}\| \oplus \bar{e}$ as a Slater point, and its dual is $\max \left\{2 \mu:-\mu\|\bar{e}\| \oplus(w+\mu \bar{e}) \in \mathrm{SOC}_{n}, \mu \in \mathbb{R}\right\}$. Since $\mu^{*}:=-\|w\|^{2} /\left(2 \bar{e}^{T} w\right)$ is optimal for the dual, it follows that

$$
2 t_{\operatorname{Diag}(w)}\left(K_{n}\right)= \begin{cases}\bar{e}^{T} w-\|w\|^{2} /\left(\bar{e}^{T} w\right) & \text { if } \bar{e}^{T} w>0 \\ 0 & \text { if } w=0 \\ -\infty & \text { otherwise }\end{cases}
$$

Now we drop the diagonal assumption, so let $W \in \mathbb{S}^{n}$ such that $\bar{e}^{T} W \bar{e}>0$ or $W \bar{e}=0$. For $y \in \mathbb{R}^{n}$, we can write $\left\langle W, y \bar{e}^{T}+\bar{e} y^{T}\right\rangle=\langle W \bar{e}, 2 y\rangle=\left\langle\operatorname{Diag}(W \bar{e}), y \bar{e}^{T}+\bar{e} y^{T}\right\rangle$, so $\left\langle W, y \bar{e}^{T}+\bar{e} y^{T}+2 I\right\rangle=\left\langle\operatorname{Diag}(W \bar{e}), y \bar{e}^{T}+\bar{e} y^{T}+2 I\right\rangle-2 \bar{e}^{T} W \bar{e}+2 \operatorname{Tr}(W)$, i.e.,

$$
4 t_{W}\left(K_{n}\right)=4 t_{\operatorname{Diag}(W \bar{e})}\left(K_{n}\right)-2 \bar{e}^{T} W \bar{e}+2 \operatorname{Tr}(W)
$$

by (5.9). Hence, (5.10) follows from (A.2). 